\title{
Buckling lengths of heavy column with various end conditions
}

\author{
Joon Kyu Lee ${ }^{a^{*}}$ and Byoung Koo Lee
}

${ }^{a}$ Department of Civil Engineering, University of Seoul, 163 Seoulsiripdae-ro, Dongdaemun-gu, Seoul 02504, South Korea ${ }^{b}$ Department of Civil and Environmental Engineering, Wonkwang University, 460 Iksan-daero, Iksan-si, Jeollabuk-do 54538, South Korea

\begin{tabular}{l}
\hline A R T I C L EI N F O \\
\hline Article history: \\
Received 25 August, 2019 \\
Accepted 23 September 2019 \\
Available online \\
23 September 2019 \\
\hline Keywords: \\
Buckling length \\
Heavy column \\
Self-weight \\
Mode shape \\
Buckling stress \\
\hline
\end{tabular}
\begin{abstract}
A B S T R A C T
This paper deals with buckling lengths of the heavy column with various end conditions, where both top and bottom ends are either free or hinged or clamped. Based on equilibrium equations of the buckled column element, the differential equation governing the buckled mode shape is derived. For solving the buckling length, the differential equation is integrated by the direct integration method and the buckling length is calculated by the determinant search method. The buckling lengths of this study agree well with those of references. The buckling lengths with various end conditions, buckled mode shapes and buckling stresses are presented.
\end{abstract}

(C) 2020 Growing Science Ltd. All rights reserved.

\section{Introduction}

In recent years, long/slender columns have often been erected for highway, bridge, marine and plant structures in practical engineering. In designs of the long column, the self-weighting effect is relatively important and must be included in buckling analyses. Such column is generally referred to as a "heavy" column. Short literature review is introduced. Firstly, Greenhill (1881) studied the stable column length, or buckling length, of the heavy column such as a vertical pole or mast in 1881. As can be seen from the title of the paper, the greatest column buckling length is compared to the maximum height at which trees considered as cantilever columns can grow. Since this paper, very little but considerable works have been done on the buckling analysis of heavy columns: Grishcoff (1930) studied buckling loads for the combined effect of both the self-weight and an axial load for the cantilever column by using an infinite series; Wang and Drachman (1981) investigated the buckling of the cantilever heavy column with an end load, based on the second order differential equation in terms of the arc length of buckled column. Interestingly, this paper included the column hanging from its fixed end, i.e., inverted cantilever column, subjected to an upward end load; Wang and Ang (1988) derived buckling formulas for the heavy column which is subjected to an axial load and is restrained by several elastic braces or internal supports; Chai and Wang (2006) determined the critical buckling load of axially compressed heavy columns with various end conditions using the differential transformation technique; Duan and Wang (2008) derived analytical exact solutions for the elastic buckling of heavy column with various of end conditions, in

* Corresponding author.

E-mail addresses: jkleegeo@uos.ac.kr (J. K. Lee) 
terms of generalized hypergeometric functions; and concerning shape optimizations of the heavy column, tallest columns with the variable cross-section and constant volume have been investigated by Keller and Niordson (1966), Atanackovic and Glavardanov (2004) and Sadiku (2008).

In spite of the considerable works discussed above, no solution methods have been found in the open literature, which are developed in this study. This study deals with the buckling lengths of the heavy column with various end conditions. Based on the small deflection beam theory, the differential equation is derived from equations of the equilibrium. As the solution methods, the direct integral method of the Runge-Kutta method for integrating the governing equation and the determinant search method enhanced by the Regula-Falsi method are developed. Predicted results of the buckling length are compared to those of references (Wang \& Ang, 1988; Duan \& Wang, 2008). The numerical results of the buckling length with the corresponding mode shapes are presented.

\section{Mathematical Formulation}

Fig. 1(a) shows the ideal column with span length $L$, vertically erected, as a plane structure, in the Cartesian Coordinates $(x, y)$ originated from the top end. The column is prismatic and the area and second moment of plane area of the cross-section are $A$ and $I$, respectively. The Young's modulus and weight density of the column material are represented as $E$ and $\gamma$, respectively. The top end is either free or hinged or clamped, and the bottom end is either hinged or clamped. Columns with the bottom free end are subjected to the tension force and thus, these columns never buckle. Such columns are out of consideration in this study. Therefore, six end conditions are possibly combined: 'free-clamped', 'hinged-hinged', 'hinged-clamped', 'clamped-hinged', 'clamped-clamped' and 'free-hinged', where the former end stands for the top end and the latter end stands for the bottom end in representation of the column end condition.

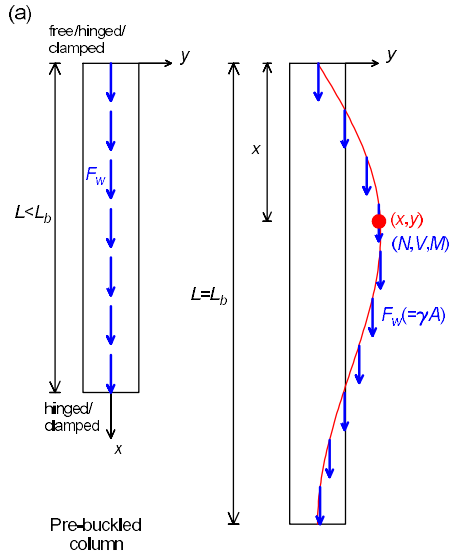

(b)

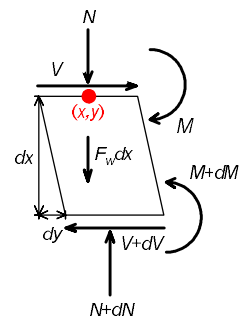

Fig. 1. (a) schematic of pre-buckled and buckled heavy column and (b) forces subjected to buckled column element.

The column is subjected to the axial compressive force due to its own self-weight. When the column length $L$ is less than the buckling length $L_{b}$, i.e., $L<L_{b}$, the column does not buckle and the column remains still straight as depicted in the pre-buckled column. However, the column length $L$ reaches $L_{b}$, i.e., $L=L_{b}$, the column, so called heavy column, buckles and forms the buckled mode shape as shown in the buckled column. In the buckled column where its mode shape is defined in the Cartesian coordinates $(x, y)$, stress resultants of the axial compressive force $N$, shear force $V$ and bending moment $M$ are subjected to the column axis. Fig. 1(b) depicts the free body diagram with the infinite length $d x$ of the buckled column element at the coordinate $x$, which is in equilibrium state from the stress resultants $(N, V, M)$ and self-weight $F_{w} d x$. Using $\sum F_{x}=0, \sum F_{y}=0$ and $\sum M=0$, three equations of the equilibrium are established as 


$$
\begin{aligned}
& \frac{d N}{d x}-F_{w}=0, \\
& \frac{d V}{d x}=0, \\
& \frac{d M}{d x}-V+N \frac{d y}{d x}=0 .
\end{aligned}
$$

Differentiate Eq. (3) with respect $x$, and substituting Eq. (1) and Eq. (2) into this result gives the following differential equation:

$$
\frac{d^{2} M}{d x^{2}}+N \frac{d^{2} y}{d x^{2}}+F_{w} \frac{d y}{d x}=0 .
$$

The self-weight intensity $F_{w}$, uniformly distributed along the column axis, due to the weight density $\gamma$ is given by

$$
F_{w}=\gamma A \text {. }
$$

Considering $F_{w}$ in Eq. (5), the axial compressive force $N$ at the coordinate $x$ is obtained as

$$
N=\int_{0}^{x} F_{w} d x=\int_{0}^{x} \gamma A d x=\gamma A x
$$

The bending moment $M$ and shear force $V$ are given by the well-known relationships between loads and deformations based on the small deflection beam theory (Gere, 2004):

$$
\begin{aligned}
& M=E I \frac{d^{2} y}{d x^{2}}, \\
& V=E I \frac{d^{3} y}{d x^{3}} .
\end{aligned}
$$

Differentiating twice Eq. (7) yields

$$
\frac{d^{2} M}{d x^{2}}=E I \frac{d^{4} y}{d x^{4}} \text {. }
$$

Substituting Eqs. (5), (6) and (9) into Eq. (4) gives the fourth order ordinary differential equation:

$$
\frac{d^{4} y}{d x^{4}}=-\frac{\gamma A}{E I}\left(x \frac{d^{2} y}{d x^{2}}+\frac{d y}{d x}\right) \text { for } 0 \leq x \leq L_{b} .
$$

To facilitate the numerical study, following non-dimensional parameters are introduced as

$$
\begin{aligned}
& \xi=\frac{x}{L_{b}}, \\
& \eta=\frac{y}{L_{b}}, \\
& \lambda_{b}=\frac{L_{b}^{3}}{E I / \gamma A},
\end{aligned}
$$

where $(\xi, \eta)$ are the non-dimensional Cartesian coordinates and $\lambda_{b}$ is the buckling length parameter. Using Eqs. (11)-(13), Eq. (10) expressed in the dimensional units is transformed into the nondimensional differential equation, Eq. (14), which governs the buckled shape of a heavy column, or

$$
\frac{d^{4} \eta}{d \xi^{4}}=-\lambda_{b}\left(\xi \frac{d^{2} \eta}{d \xi^{2}}+\frac{d \eta}{d \xi}\right) \text { for } 0 \leq \xi \leq 1,
$$


where $\lambda_{b}$ is the eigenvalue in Eq. (14), which will be calculated in this study. Now, consider boundary conditions for Eq. (14). At the top free end $(x=0), M$ (Eq. (7)) and $V$ (Eq. (8)) are zero 0. Thus, the non-dimensional boundary conditions of the top free end $(\xi=0)$ are obtained as

$$
\frac{d^{2} \eta}{d \xi^{2}}=0 ; \frac{d^{3} \eta}{d \xi^{3}}=0
$$

For the top and bottom hinged ends $\left(x=0\right.$ and $\left.x=L_{b}\right), y$ and $M$ are zero, and the non-dimensional boundary conditions at $\xi=0$ and $\xi=1$ are obtained as follows.

$$
\eta=0 ; \frac{d^{2} \eta}{d \xi^{2}}=0
$$

For the top and bottom clamped ends $\left(x=0\right.$ and $\left.x=L_{b}\right), y$ and $d y / d x$ are zero, and the nondimensional boundary conditions at $\xi=0$ and $\xi=1$ are obtained as follows.

$$
\eta=0 ; \frac{d \eta}{d \xi}=0 .
$$

\section{Solution Methods}

Based on the above analysis, a general Fortran computer program was written to calculate the buckling length parameter $\lambda_{b}$ and its corresponding buckled mode shape $(\xi, \eta)$. The differential equation, Eq. (14), is the boundary and eigenvalue problems. For computing the mode shape $(\xi, \eta)$, the Eq. (14) as a boundary problem subjected to end conditions selected from Eqs. (15)-(17) is integrated numerically using the direct integration method such as Runge-Kutta method (Burden et al, 2016) and the eigenvalue $\lambda_{b}$ is computed by the determinant search method (Lee \& Lee, 2019; Lee et al., 2019) enhanced by Regula-Falsi method (Burden et al., 2016). Using these solution methods, $\lambda_{b}$ with the mode shapes $(\xi, \eta)$ of the various end conditions are calculated, in which six end conditions are considered, mentioned previously in Section 2. These kinds of solution methods for the boundary and eigenvalue problems like Eq. (14) are described in detail in references (Lee \& Lee, 2018, 2019; Lee et al., 2019). In this study, the lowest, i.e., first, value of $\lambda_{b}$ is computed. It is important to obtain the suitable step size $\Delta \xi$ in the RungeKutta method, which is calculated from the following equation for a given number of dividing element $n$ of the unit column length.

$$
\Delta \xi=\frac{1}{n} \text {. }
$$

Accordingly, the convergence analysis was performed to obtain the suitable $n(=1 / \Delta \xi)$ and the result of the free-clamped column is shown in Fig. 2. It is observed that the $\lambda_{b}$ solution with $n=30$ agrees well with the solution with $n=200$ to within five significant figures. All computations were carried out on a PC with graphics support, and $\lambda_{b}$ with the mode shape $(\xi, \eta)$ for $n=100$ was computed within one third second.

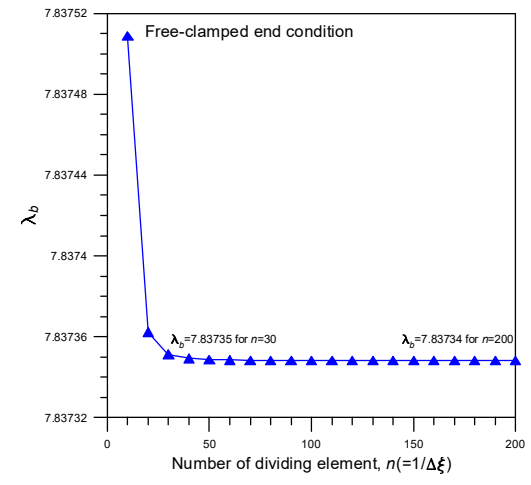

Fig. 2. Convergence analysis for suitable $n(=1 / \Delta \xi)$ in Runge-Kutta method. 


\section{Results and Discussion}

The buckling length parameter $\lambda_{b}$ by end condition obtained from this study are presented in Table 1 in which those obtained from references (Wang \& Ang, 1988; Duan \& Wang, 2008) are listed for the validation purpose. The results of this study and Duan and Wang (2008) are identical to the six figures accuracy, and results of Wang and Ang (1988) agree well within 5.8\% error. It can be observed that $\lambda_{b}$ depends significantly on the end condition. For example, $\lambda_{b}$ of a clamped-clamped column is 9.52 times longer than $\lambda_{b}$ of a free-clamped column. Interestingly, the free-hinged column theoretically buckles under $\lambda_{b}=25.6381$ even though this column is structurally unstable. Comparing results between hinged-clamped and clamped-hinged columns, it is advantageous to place the end with higher degree of freedom on the bottom to obtain a longer $\lambda_{b}$.

Table 1. Buckling length parameter $\lambda_{b}$ by end condition with those of references.

\begin{tabular}{lllllll}
\hline End condition & Free - clamped & Hinged - hinged & Hinged - clamped & Clamped - hinged & Clamped - clamped & Free - hinged \\
\hline Present study & 7.83734 & 18.5687 & 52.5007 & 30.0094 & 74.6286 & 25.6381 \\
Duan and Wang (2008) & 7.8373 & 18.5687 & 52.5007 & - & 74.6286 & - \\
Wang and Ang (1988) & 7.84 & 18.58 & 53.91 & - & 78.96 & - \\
\hline
\end{tabular}

Fig. 3 shows buckled mode shapes by end condition. It is noted that even though each end condition has the different value of $\lambda_{b}$, all horizontal column axes are unified as unity $1\left(=L_{b} / L_{b}\right)$ because the non-dimensional coordinate $\xi$ is normalized by the buckling length $L_{b}$ regardless of the end condition (see Eq. (11)). The location of maximum deflection of buckled column depends on the end condition. The location of maximum deflection of column like the utility pole may be supported by the guy-wires to protect the unexpected buckling from undesirable column imperfection.
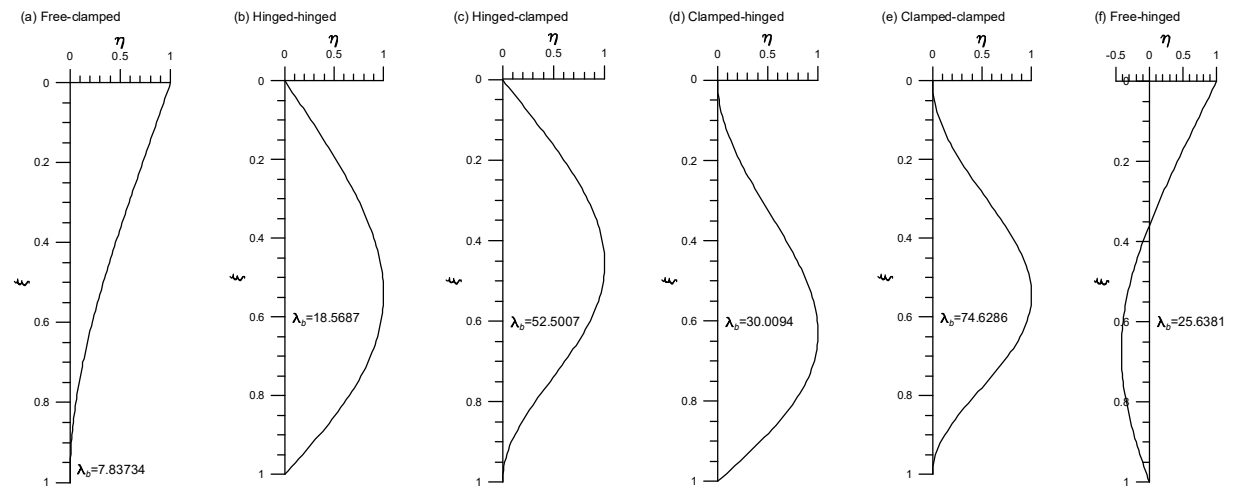

Fig. 3. Buckled mode shapes by end condition

For a given set of column parameters of $E, \gamma, A$ and $I$, the buckling length $L_{b}$ and buckling (maximum) stress $\sigma_{b}$ occurred at the bottom end $\left(x=L_{b}\right)$ can be obtained from $\lambda_{b}$ presented in Table 1:

$$
\begin{aligned}
L_{b} & =\sqrt[3]{\frac{E I}{\gamma A} \lambda_{b}}, \\
\sigma_{b} & =\frac{W}{A}=\frac{\gamma A L_{b}}{A}=\gamma L_{b},
\end{aligned}
$$

where $W\left(=\gamma A L_{b}\right)$ is the column weight. For showing numerical examples of $L_{b}$ and $\sigma_{b}$, two practical heavy columns are considered: (a) Wooden solid circular cross-section with the radius $r=0.1 \mathrm{~m}, E=$ $13 \mathrm{GPa}, \gamma=5 \mathrm{kN} / \mathrm{m}^{3}$ (then, $A=0.03142 \mathrm{~m}^{2}, I=7.854 \mathrm{e}-5 \mathrm{~m}^{4}$ and $L_{b}=18.66 \sqrt[3]{\lambda_{b}} \mathrm{~m}$ ); and (b) Steel hollow circular cross-section with the outer radius $r=0.1 \mathrm{~m}$, thickness $=0.02 \mathrm{~m}, E=210 \mathrm{GPa}$ and $\gamma=77 \mathrm{kN} / \mathrm{m}^{3}$ (then, $A=1.244 \mathrm{e}-3 \mathrm{~m}^{2}, I=6.097 \mathrm{e}-6 \mathrm{~m}^{4}$ and $L_{b}=23.73 \sqrt[3]{\lambda_{b}} \mathrm{~m}$ ). The results by end condition are presented in Table 2 . In the practical engineering field, the column length of the freeclamped column, which is often erected for the utility poles, can rise by about $37 \mathrm{~m}$ for the wooden pole and about $47 \mathrm{~m}$ for the steel pole. Considering yield stresses $\sigma_{y}=50 \mathrm{MPa}$ for wood and $\sigma_{y}=1,000$ 
$\mathrm{MPa}$ for steel, values of $\sigma_{b}$ are relatively very small compared to $\sigma_{y}$, and it is clear that heavy columns rupture to the buckling rather than the crushing.

Table 2. Buckling length $L_{b}$ and buckling stress $\sigma_{b}$ by end condition.

\begin{tabular}{llllll}
\hline (a) Wooden solid circular cross-section & & & \\
\hline$L_{b}$ and $\sigma_{b}$ & Free - clamped & Hinged - hinged & Hinged - clamped & Clamped - hinged & Clamped - clamped \\
\hline$L_{b}(\mathrm{~m})$ & 37.07 & 49.41 & 69.87 & 57.99 & 78.56 \\
$\sigma_{b}(\mathrm{MPa})$ & 0.1854 & 0.2471 & 0.3494 & 0.2900 & 0.3928 \\
\hline (b) Steel hollow circular cross-section & & & & 9 \\
\hline$L_{b}(\mathrm{~m})$ & 47.14 & 62.84 & 88.86 & 73.74 & 79.91 \\
$L_{b}(\mathrm{MPa})$ & 3.630 & 4.839 & 6.842 & 5.678 & 7.693 \\
\hline
\end{tabular}

\section{Concluding Remarks}

This paper presents the buckling lengths of the heavy column with various end conditions. Using equilibrium equations of the buckled column element based on the small deflection beam theory, the fourth order ordinary differential equation governing the buckled mode shape is derived. For solving the differential equation, both the direct integration method and determinant search method are developed as the solution methods. The predicted buckling lengths compare well with those of references (Wang \& Ang, 1988; Duan \& Wang, 2008). The buckling lengths with various end conditions, buckled mode shapes and buckling stresses are presented. In further study, the theory of this study should be combined with the end bearing long slender piles embedded in the soil foundation.

\section{References}

Atanackovic, T. M., \& Glavardanov, V. B. (2004). Optimal shape of a heavy compressed column. Structural and Multidisciplinary Optimization, 28(6), 388-396.

Burden, R.L., Faires, D.J. \& Burden, A.M. (2016). Numerical Analysis. Cengage Learning, MA, USA.

Chai, Y. H., \& Wang, C. M. (2006). An application of differential transformation to stability analysis of heavy columns. International Journal of Structural Stability and Dynamics, 6(03), 317-332.

Duan, W. H., \& Wang, C. M. (2008). Exact solution for buckling of columns including self-weight. Journal of Engineering Mechanics, 134(1), 116-119.

Gere, J.M. (2004). Mechanics of Materials. Brooks/Cole-Thomson Learning, CA, USA.

Greenhill, A.G. (1881). Determination of the greatest height consistent with stability that a vertical pole or mast can be made, and the greatest height to which a tree of given proportions can grow. Mathematical Proceedings of the Cambridge Philosophical Society, 65-73.

Grishcoff, N. (1930). Theory of elastic stability. Bull. Aca. Sci. Kiev., 101.

Keller, J.B., \& Niordson, F.I. (1966). The tallest column. Journal of Mathematical Mechanics, 165, 433-446.

Lee, J.K., \& Lee, B.K. (2019). Free vibration and buckling of tapered columns made of axially functionally graded materials. Applied Mathematical Modeling, 75, 73-87.

Lee, J., \& Lee, B. (2018). Elastica and buckling loads of nonlinear elastic tapered cantilever columns. Engineering Solid Mechanics, 6(1), 39-50.

Lee, J. K., Park, S. H., \& Kim, Y. (2019). Transverse free vibration of axially loaded tapered friction piles in heterogeneous soil. Soil Dynamics and Earthquake Engineering, 117, 116-121.

Sadiku, S. (2008). Buckling load optimization for heavy elastic columns: a perturbation approach. Structural and Multidisciplinary Optimization, 35(5), 447-452.

Wang, C. M., \& Ang, K. K. (1988). Buckling capacities of braced heavy columns under an axial load. Computers \& Structures, 28(5), 563-571.

Wang, C. Y., \& Drachman, B. (1981). Stability of a heavy column with an end load. Journal of Applied Mechanics, 48(3), 668-669.

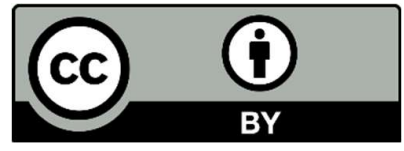

(C) 2020 by the authors; licensee Growing Science, Canada. This is an open access article distributed under the terms and conditions of the Creative Commons Attribution (CC-BY) license (http://creativecommons.org/licenses/by/4.0/). 